\title{
Edge-Magic Total Labellings of Some Network Models
}

\author{
Hongyu WANG, Bing YAO*, Chao YANG, Sihua \\ YANG, Xiang'en CHEN \\ College of Mathematics and Statistics, Northwest \\ Normal University, Lanzhou, 730070, China \\ E-mail: yybb918@163.com
}

\author{
Ming Yao, Zhenxue Zhao \\ Department of Information Process and Control \\ Engineering, Lanzhou Petrochemical College of \\ Vocational Technology, Lanzhou, 730060, \\ e-mail: yybm918@163.com
}

\begin{abstract}
It has been known that edge-symmetric graphs can be used as models of some scale-free networks, such as hierarchial networks and self-similar networks, such as graph colorings can be used for distinguishing objects of communication and informa-tion networks. We study the edgemagic property of edge-symmetric graphs, and construct graphs having edge-magic total labellings from smaller graphs.
\end{abstract}

Keywords-network; tree; set-ordered labellings; edge-magic total labelling; edge-symmetric graphs

\section{INTRODUCTION}

Graph coloring theory is one of the most actively branch in graph theory. It involves in many fields, such as such as physics, chemistry, computer science, network theory, social science, etc. And graph labelings provide useful mathematical models for a wide range of applications, such as data security, cryptography (secret sharing schemes), astronomy, various coding theory problems, communication networks, mobile telecommunication systems, bioinformatics and X-ray crystallography. More detailed discussions on applications of graph labelings can be found in Bloom and Golomb's papers [11] and [12]. Many studies in graph labeling refer to Rosa's research [13].

The mathematical model of scale-free networks in study of complex networks is closed to real networks. It has been known that edge-symmetric graphs can be used as models of some scale-free networks, such as hierarchial networks and self-similar networks, etc. Li et al. [18] critically overviews the current understanding on scale-freeness and proposes its mathematically rigorous definition of scale-free graphs. Yao et al. [19] present: The notation $N(t)=(p(u, k, t), G(t))$ denotes a dynamic network, where $p(u, k, t)$ is the probability such that the probability of a new node being adjacent to $k$ other nodes submits to $p(u, k, t), G(t)$ is the connected topological structure (also, graph) of $N(t), t \in[a$, $b]$; $G(a)$ is the initially connected graph of $N(t)$ at $t=a$. We say a node of $N(t)$ an alltime-hub node if it is not a leaf of any spanning tree $T(t)$ having maximal leaves in $G(t)$ for each time $t \in[a, b]$. By the method of analyzing spanning trees in scale-free networks, some problems are researched in [20]. As a result, finding a spanning tree with as many leaves as possible (MLATP is one of the classical NPcomplete problems) is equal to finding a minimal connected dominating set in a connected network. It is very important to make network models for simulating real networks.

Sedlacek [14] published a paper about another kind of graph labeling, called the labeling magic. His definition was motivated by the magic square notion in number theory. A magic labeling is a function from the set of edges of a graph $G$ into the non-negative real numbers, so that the sums of the edge labels around any vertex in $G$ are all the same. Stewart [15] called magic labeling supermagic if the set of edge labels consisted of consecutive integers. Motivated by Sedlacek and Stewart's research, many new related definitions have been proposed and new results have been found.

Conjecture 1. [16] Every tree admits an edge-magic total labelling.

Conjecture 2. [17] Every tree admits a super edgemagic total labelling.

An edge-symmetric graph $H$ is a connected graph having a non-empty proper subset $S \subset E(H)$ such that $H-S$ contains $m(\geq 2)$ components $H_{1}, H_{2}, \cdots, H_{m}$ with $H_{i} \cong H_{j}$. It has been known that edge-symmetric graphs can be used as models of researching scale-free networks, hierarchical networks and self-similar networks [2]. We use standard notation and terminology of graph theory here. The graphs mentioned are finite graphs without loops, multiple or directed edges. The shorthand notation $[m, n]$ stands for an integer set $\{m, m+1, \cdots, n\}$, where $n>m \geq 0$. A $(p, q)$-graph $G$ is one with $p$ vertices and $q$ edges.

Definition 1. [3] If a $(p, q)$-graph $G$ admits a mapping $f: V(T)[0, q]$ such that $f(u) \neq f(v)$ for distinct $u, v \in V(G)$, and the edge label set $\{f(u v)=|f(u)-f(v)|: u v \in E(G)\}=[1, q]$, then $f$ is called a graceful labelling, also, $G$ is graceful.

We write $f(V(G))=\{f(u): u \in V(G)\}$ and $f(E(G))=\{f(u v)$ : $u v \in E(G)\}$ hereafter.

Definition 2. ([7],[9]) A bipartite graph $G$ admits a graceful labelling $f$. If $\max \{f(x): x \in X\}<\min \{f(y): y \in Y\}$, where $(X, Y)$ is the bipartition of $V(G)$, then $f$ is called a setordered graceful labelling, and this case is denoted as $f(X)<f(Y)$.

Definition 3. ([3],[5]) Let $G$ be a $(p, q)$-graph. If there exists a constant $\lambda$ and a bijection $f: V(G) \cup E(G) \rightarrow[1, p+q]$ such that $f(u)+f(v)+f(u v)=\lambda$ for every edge $u v \in E$, then we say $f$ an edge-magic total labelling of $G$, and $\lambda$ a magic constant. Furthermore, if $G$ is a bipartite graph with bipartition $(X, Y)$, and $f$ holds $f(V(G))=[1, p]$ and $\max \{f(x)$ : 
$x \in X\}<\min \{f(y): y \in Y\}$ (denoted as $f(X)<f(Y)$ ), we call $f$ a super set-ordered edge-magic total labelling.

Definition 4. Let $T$ be a tree having vertex set $V(T)=X \cup$ $Y$, where $X=\left\{w_{1}, w_{2}, \cdots, w_{l}\right\}, Y=\left\{w_{l+1}, w_{l+2}, \cdots, w_{p}\right\}$, and let $H_{k}$ be a tree with the bipartition $\left(X_{k}, Y_{k}\right)$ for $k \in[1, p]$. A uniformly edge-symmetric tree $G=\left\langle T ; H_{1}, H_{2}, \cdots, H_{p}\right\rangle$ is a tree obtained by identifying $w_{i} \in V(T)$ with a vertex $v_{i} \in V\left(H_{i}\right)$ for $i \in[1, p]$, whenever $\left|X_{i}\right|=\left|X_{j}\right|$ and $\left|Y_{i}\right|=\left|Y_{j}\right|$ for $j \neq i$.

\section{MAIN RESULTS}

Theorem 1. Let $T$ be a set-ordered graceful tree on $p$ vertices, and its bipartition $(X, Y)$ hold ||$X|-| Y|| \leq 1$. Suppose that every $H_{k}$ admits super set-ordered edge-magic total labellings for $k \in[1, p]$. Then the uniformly edge-symmetric tree $\left\langle T ; H_{1}, H_{2}, \cdots, H_{p}\right\rangle$ admits super set-ordered edge-magic total labellings.

Theorem 2. Let $T$ be a tree on $p$ vertices, and its bipartition $(X, Y)$. Suppose that every $H_{k}$ admits super setordered edge-magic total labellings for $k \in[1, p]$, the $V\left(X_{i}\right)+V\left(X_{p-i}\right)=m, \quad V\left(Y_{i}\right)+V\left(Y_{p-i}\right)=m$, and $V\left(X_{\delta}\right)=V\left(Y_{\delta}\right)$ for $\delta=\lfloor p / 2\rfloor$ and $m \in N^{+}, i \in[1, \delta]$. Then the uniformly edgesymmetric tree $\left\langle T ; H_{1}, H_{2}, \cdots, H_{p}\right\rangle$ admits super set-ordered edge-magic total labellings.

Corollary 3. Let $T$ be a set-ordered graceful tree on $p$ vertices, and its bipartition $(X, Y)$ hold $\| X|-| Y|| \leq 1$. Suppose that every $H_{k}$ admits set-ordered graceful labellings for $k \in$ $[1, p]$. Then the uniformly edge-symmetric tree $\left\langle T ; H_{1}, H_{2}, \cdots\right.$, $\left.H_{p}\right\rangle$ admits super set-ordered edge-magic total labellings.

Corollary 4. Let $T$ be a set-ordered graceful tree on $p$ vertices, and its bipartition $(X, Y)$ hold $\| X|-| Y|| \leq 1$. Suppose that every $H_{k}$ admits set-ordered graceful labellings for $k \in[1, p]$. Then the uniformly edge-symmetric tree $\left\langle T ; H_{1}\right.$, $\left.H_{2}, \cdots, H_{p}\right\rangle$ admits set-ordered graceful labellings.

Corollary 5. Let $T$ be a set-ordered graceful tree on $p$ vertices, and its bipartition $(X, Y)$ hold $\| X|-| Y|| \leq 1$. Suppose that every $H_{k}$ admits super set-ordered edge-magic total labellings for $k \in[1, p]$. Then the uniformly edge-symmetric tree $\left\langle T ; H_{1}, H_{2}, \cdots, H_{p}\right\rangle$ admits set-ordered graceful labellings.

Corollary 6. Let $T$ be a super set-ordered edge-magic total tree on $p$ vertices, and its bipartition $(X, Y)$ hold $\| X|-| Y|| \leq 1$. Suppose that every $H_{k}$ admits super set-ordered edge-magic total labellings for $k \in[1, p]$. Then the uniformly edge-symmetric tree $\left\langle T ; H_{1}, H_{2}, \cdots, H_{p}\right\rangle$ admits super setordered edge-magic total labellings.

Corollary 7. Let $T$ be a super set-ordered edge-magic total tree on $p$ vertices, and its bipartition $(X, Y)$ hold $\| X|-| Y|| \leq 1$. Suppose that every $H_{k}$ admits super set-ordered edge-magic total labellings for $k \in[1, p]$. Then the uniformly edge-symmetric tree $\left\langle T ; H_{1}, H_{2}, \cdots, H_{p}\right\rangle$ admits set-ordered graceful labellings.

Corollary 8. Let $T$ be a super set-ordered edge-magic total tree on $p$ vertices, and its bipartition $(X, Y)$ hold $\| X|-| Y|| \leq 1$. Suppose that every $H_{k}$ admits set-ordered graceful labellings for $k \in[1, p]$. Then the uniformly edge- symmetric tree $\left\langle T ; H_{1}, H_{2}, \cdots, H_{p}\right\rangle$ admits super set-ordered edge-magic total labellings.

Corollary 9. Let $T$ be a super set-ordered edge-magic total tree on $p$ vertices, and its bipartition $(X, Y)$ hold ||$X|-| Y|| \leq 1$. Suppose that every $H_{k}$ admits set-ordered graceful labellings for $k \in[1, p]$. Then the uniformly edgesymmetric tree $\left\langle T ; H_{1}, H_{2}, \cdots, H_{p}\right\rangle$ admits set-ordered graceful labellings.
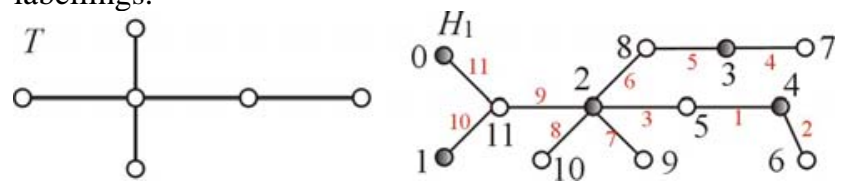

Figure-1. $\quad T$ and $H_{1}$ for Theorem 2.

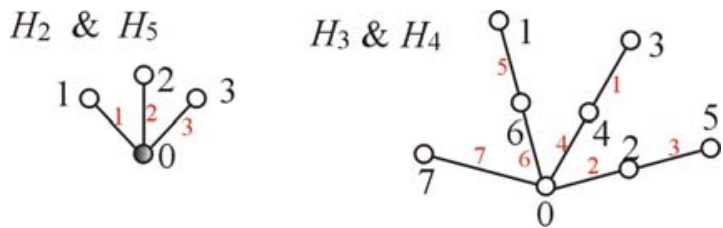

Figure-2. $H_{2}, H_{5}$ and $H_{3}, H_{4}$ for Theorem 2.

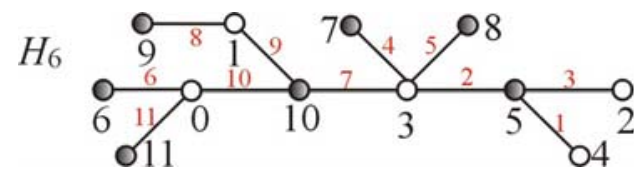

Figure-3. $H_{6}$ for Theorem 2.

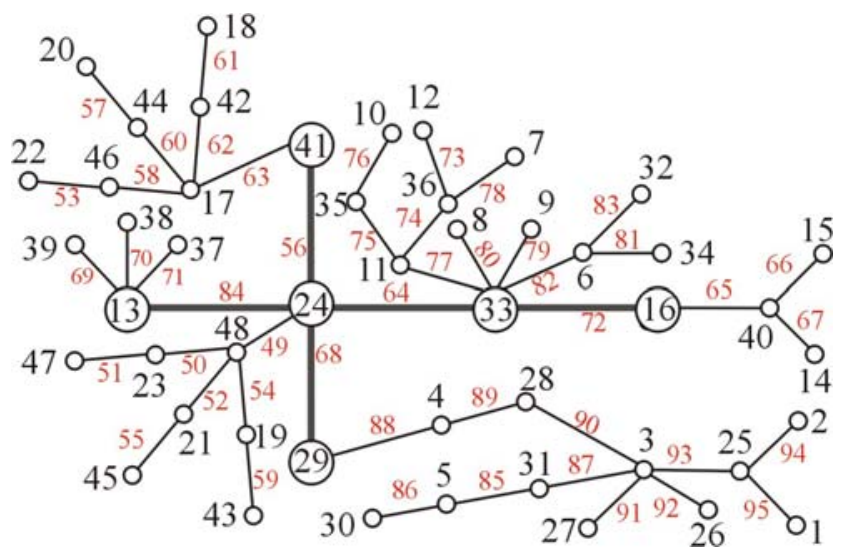

Figure-4. An example $G=\left\langle T ; H_{1}, H_{2}, \cdots, H_{6}\right\rangle$ for Theorem 2 based on Figure-1, Figure-2 and Figure-3.

\section{PROOFS}

Proof of Theorem 1. Use the notations of Definition 4 for $G=\left\langle T ; H_{1}, H_{2}, \cdots, H_{p}\right\rangle$. Let $(X, Y)$ be the bipartition of a set-ordered graceful tree $T$, where $\| X|-| Y|| \leq 1, X=\left\{w_{1}, w_{2}, \cdots\right.$, $\left.w_{l}\right\}$ and $Y=\left\{w_{l+1}, w_{l+2}, \cdots, w_{p}\right\}$. By this theorem's hypothesis, $T$ has a set-ordered graceful labelling $f$ such that $f\left(w_{i}\right)=i-1$, $i \in[1, p]$, since $f(X)<f(Y)$.

Notice that $G$ is a uniformly edge-symmetric tree. By Definition 4 we can prove the following properties of $G$.

P1. Every tree $H_{k}$ has the same number $\left|H_{k}\right|=n$ from $s=\left|X_{k}\right|=\left|X_{j}\right|$ and $t=\left|Y_{k}\right|=\left|Y_{j}\right|$ for $j \neq k$. 
P2. Every tree $H_{k}$ admits a super set-ordered edge-magic total labelling $g_{k}$ such that $g_{k}\left(u_{k, 1}\right)<g_{k}\left(u_{k, 2}\right)<\cdots<g_{k}\left(u_{k, s}\right)<$ $g_{k}\left(v_{k, t}\right)<g_{k}\left(v_{k, t-1}\right)<\cdots<g_{k}\left(v_{k, 1}\right)$, for $s+t=n, u_{k, i} \in X_{k}=\left\{u_{k, i}: i \in[1\right.$, $s]\}, v_{k, j} \in Y_{k}=\left\{v_{k, j}: j \in[1, t]\right\}$ for $k \in[1, p]$.

P3. Every edge $u_{k, i} v_{k, j}$ of each tree $H_{k}$ holds $g_{k}\left(u_{k, i}\right)+$ $g_{k}\left(u_{k, i} v_{k, j}\right)+g_{k}\left(v_{k, j}\right)=a$ (because $g_{k}$ is every $H_{k}$ admits super setordered edge-magic total labellings for $k \in[1, p])$, for $i \in[1$, $s], j \in[1, t]$ and $k \in[1, p]$.

P4. Two trees $H_{i}$ and $H_{j}$ both have the same label sets $g_{k}\left(V\left(H_{k}\right)\right)=[1, n], g_{k}\left(E\left(H_{k}\right)\right)=[n+1,2 n-1]$ for $k \in[1, p]$.

We can take a tree $H_{0}$ as a representative of $H_{1}, H_{2}, \cdots$, $H_{p}$, where $V\left(H_{0}\right)=X_{0} \cup Y_{0}$ with $X_{0}=\left\{u_{i}: i \in[1, s]\right\}$ and $Y_{0}=\left\{v_{j}\right.$ : $j \in[1, t]\}$. Furthermore, $H_{0}$ admits a super set-ordered edgemagic total labelling $g_{0}$ such that $g_{0}\left(u_{i}\right)<g_{0}\left(u_{i+1}\right)$ for $i \in[1, s-1], g_{0}\left(u_{s}\right)<g_{0}\left(v_{1}\right)$, and $g_{0}\left(v_{j}\right)<g_{0}\left(v_{j+1}\right)$ for $j \in[1, t-1]$. According to the above deduction, we have $g_{k}\left(u_{k, i}\right)=g_{0}\left(u_{i}\right)=i$ for $u_{k, i} \in X_{k}$ and $u_{i} \in X_{0}, g_{k}\left(v_{k, j}\right)=g_{0}\left(v_{t+1-j}\right)=n+1-j$ for $v_{k, j} \in Y_{k}$, $v_{j} \in Y_{0}, g_{k}\left(u_{k, i} v_{k, j}\right)=g_{0}\left(u_{k, i} v_{t+1-j}\right)=2 n-t+j-i$ and $k \in[1, p]$. From $g_{0}\left(V\left(H_{i}\right)\right)=[1, n]$ and $g_{k}\left(E\left(H_{k}\right)\right)=[n+1,2 n-1]$, we obtain $g_{k}\left(u_{k, i}\right)+g_{k}\left(u_{k, i} v_{k, j}\right)+g_{k}\left(v_{k, j}\right)=g_{0}\left(u_{1}\right)+g_{0}\left(v_{1}\right)+2 n-1$, for $i \in[1, s]$, $j \in[1, t]$ and $k \in[1, p]$.

We use the labelling $f$ of $T$ to define another labelling $f^{\prime}$ of $T$ as: $f^{\prime}\left(w_{i}\right)=f\left(w_{i}\right)+1, \mathrm{i} \in[1, p], f^{\prime}\left(w_{i} w_{j}\right)=n\left|f\left(w_{i}\right)-f\left(w_{j}\right)\right|+p n$.

By the parity of $p$, we define a labelling $g$ of the uniformly edge-symmetric tree $G$ in the following.

Case 1. If $p=2 \beta+1$, thus $\| X|-| Y|| \leq 1$.

Let $\lambda=(5 \beta+2) n+g_{0}\left(v_{1}\right)$. The labelling $g$ of $G$ can be defined as: (i) When $k \in[1, \beta+1]$, set $g\left(u_{k, i}\right)=n(k-1)+1+$ $g_{0}\left(u_{k}\right)-g_{0}\left(u_{1}\right), i \in[1, s] ; g\left(v_{k, j}\right)=n(\beta+k-1)+g_{0}\left(v_{j}\right), j \in[1, t]$; and for each edge $u_{k, i} v_{k, j} \in E(G)$, let $g\left(u_{k, i} v_{k, j}\right)=g_{0}\left(u_{k, i} v_{k, j}\right)+$ $2 n(2 \beta+1-k), k \in[1, \beta+1]$.

(ii) When $l \in[\beta+2,2 \beta+1]$, set $g\left(v_{l, j}\right)=n(2 \beta+2-l)-g_{0}\left(v_{j}\right)+$ $g_{0}\left(v_{1}\right), \quad j \in[1, t] ; \quad g\left(u_{l, i}\right)=n(3 \beta+2-l)+g_{0}\left(v_{1}\right)-1-g_{0}\left(u_{i}\right)+g_{0}\left(u_{1}\right)$, $i \in[1, s]$; and for every edge $u_{l, i} v_{l, j} \in E(G)$, set $g\left(u_{l, i} v_{l, j}\right)=2 l n$ $-g_{0}\left(u_{i} v_{j}\right)$. For $k \in[1, \beta+1]$ and $l \in[\beta+2,2 \beta+1]$ as well as $k+l=2(\beta+1)$, we have $g\left(u_{k, i}\right)+g\left(u_{l, j}\right)=n(k-1)+n(3 \beta+2-l)+$ $g_{0}\left(v_{1}\right)=\lambda-n(2 l-1), g\left(v_{k, i}\right)+g\left(v_{l, j}\right)=n(\beta+k-1)+n(2 \beta+2-l)+g_{0}\left(v_{1}\right)$ $=\lambda-n(2 l-1), g\left(u_{k, i} v_{k, j}\right)+g\left(u_{l, i} v_{l, j}\right)=2 n(2 \beta+1-k)+2 \ln =2 n(2 l-1)$.

The above show that for $k+l=2(\beta+1), H_{k}$ and $H_{l}$ form a matchable pair. Next, our aim is to prove that $g$ is a super set-ordered edge-magic total labelling of $G$. For $k \in[1, \beta+1]$, $i \in[1, s]$ and $j \in[1, t]$, each edge $u_{k, i} v_{k, j} \in E\left(H_{k}\right)$ holds $g\left(u_{k, i}\right)+$ $g\left(u_{k, i} v_{k, j}\right)+g\left(v_{k, j}\right)=n(5 \beta+2)+g_{0}\left(v_{1}\right)=\lambda$. For $l \in[\beta+2,2 \beta+1]$, $i \in[1, s]$ and $j \in[1, t]$, every edge $u_{l, i} v_{l, j} \in E\left(H_{l}\right)$ holds $g\left(u_{l, i}\right)+$ $g\left(u_{l, i} v_{l, j}\right)+g\left(v_{l, j}\right)=n(5 \beta+2)+g_{0}\left(v_{1}\right)=\lambda$. So, $g$ is a labelling of $H_{k}$ such that $g\left(u_{k, i}\right)+g\left(u_{k, i} v_{k, j}\right)+g\left(v_{k, j}\right)=\lambda$ for $k \in[1,2 \beta+1]$.

For $k \in[1,2 \beta+1]$ and $i \in[1, s]$, if we identify the vertex $u_{k, i} \in X_{k} \subseteq V\left(H_{k}\right)$ with the vertex $w_{k} \in V(T)$ into one vertex, and then substitute $f^{\prime}\left(w_{k}\right)$ by $g\left(u_{k, i}\right)$. Finally, set $g\left(w_{i} w_{j}\right)=f^{\prime}\left(w_{i} w_{j}\right)$ for edges $w_{i} w_{j} \in E(T)$. For $k \in[1,2 \beta+1]$ and $j \in[1, t]$, if we identify the vertex $v_{k, j} \in Y_{k} \subseteq V\left(H_{k}\right)$ with the vertex $w_{k} \in V(T)$ into one, we replace $f^{\prime}\left(w_{k}\right)$ by $g\left(v_{k, j}\right)$, and then define $g\left(w_{i} w_{j}\right)=f^{\prime}\left(w_{i} w_{j}\right)$ for edges $w_{i} w_{j} \in E(T)$. Here, the labelling $g$ of $G$ is defined well.

We verify that $f^{\prime}\left(w_{i}\right)+f^{\prime}\left(w_{i} w_{j}\right)+f^{\prime}\left(w_{j}\right)$ is equal to a constant for every edge $w_{i} w_{j} \in E(T)$.

(1.1) For a fixed $i_{0} \in[1, s]$, we identify the vertex $u_{k, i_{0}} \in X_{k} \subseteq V\left(H_{k}\right)$ with the vertex $w_{k} \in V(T)$ into one vertex. For $i \in[1, \beta+1], j \in[\beta+2,2 \beta+1]$ and $l \in[1, s]$, every edge $w_{i} w_{j} \in E(T) \subseteq E(G)$ satisfies $f^{\prime}\left(w_{i}\right)+f^{\prime}\left(w_{i} w_{j}\right)+f^{\prime}\left(w_{j}\right)=g\left(u_{i, l}\right)+$ $g\left(w_{i} w_{j}\right)+g\left(u_{j, l}\right)=n(i-1)+1+g_{0}\left(u_{l}\right)-g_{0}\left(u_{1}\right)+n\left|f\left(w_{i}\right)-f\left(w_{j}\right)\right|+n(2 \beta+$ $1)+n(3 \beta+2-j)+g_{0}\left(v_{1}\right)-1-g_{0}\left(u_{l}\right)+g_{0}\left(u_{1}\right)=n(i+5 \beta+2-j)+n(j-i)+$ $g_{0}\left(v_{1}\right)=n(5 \beta+2)+g_{0}\left(v_{1}\right)=\lambda$.

(1.2) For a fixed $j_{0} \in[1, t]$, we identify the vertex $v_{k, j_{0}} \in Y_{k} \subseteq V\left(H_{k}\right)$ with the vertex $w_{k} \in V(T)$ into one vertex. For $i \in[1, \beta+1], j \in[\beta+2,2 \beta+1]$ and $l \in[1, t]$, each edge $w_{i} w_{j} \in E(T) \subseteq E(G)$ satisfies $f^{\prime}\left(w_{i}\right)+f^{\prime}\left(w_{i} w_{j}\right)+f^{\prime}\left(w_{j}\right)=g\left(v_{i, l}\right)+$ $g\left(w_{i} w_{j}\right)+g\left(v_{j, l}\right)=g\left(v_{i, l}\right)+n\left|f\left(w_{i}\right)-f\left(w_{j}\right)\right|+n(2 \beta+1)+g\left(v_{j, l}\right)=n(\beta+i-1$ )$+g_{0}\left(v_{l}\right)+n\left|f\left(w_{i}\right)-f\left(w_{j}\right)\right|+n(2 \beta+1)+n(2 \beta+2-j)-g_{0}\left(v_{l}\right)+g_{0}\left(v_{1}\right)=n($ $5 \beta+2)+g_{0}\left(v_{1}\right)=\lambda$.

The above deduction shows that the bipartition $\left(X^{*}, Y^{*}\right)$ of $G$ holds $g\left(X^{*}\right)<g\left(Y^{*}\right)$, where $g\left(X^{*}\right)=\left\{g\left(u_{k, i}\right): k \in[1, \beta+1]\right.$, $i \in[1, s]\} \cup\left\{g\left(v_{k, j}\right): k \in[\beta+2,2 \beta+1], j \in[1, t]\right\}, g\left(Y^{*}\right)=\left\{g\left(u_{k, i}\right)\right.$ : $k \in[\beta+2,2 \beta+1], i \in[1, s]\} \cup\left\{g\left(v_{k, j}\right): k \in[1, \beta+1], j \in[1, t]\right\}$.

The edge label set $g(E(G)=[n(2 \beta+1)+1,2 n(2 \beta+1)-1]$.

(1) For every edge $u_{k, i} v_{k, j} \in E(G)$ with $k \in[1,2 \beta+1], i \in[1$, $s]$ and $j \in[1, t]$, then $g\left(u_{k, i}\right)+g\left(u_{k, i} v_{k, j}\right)+g\left(v_{k, j}\right)=\lambda$; (2) For every edge $w_{i} w_{j} \in E(T)$ with $i \in[1, \beta+1]$ and $j \in[\beta+2,2 \beta+1]$, then $f^{\prime}\left(w_{i}\right)+f^{\prime}\left(w_{i} w_{j}\right)+f^{\prime}\left(w_{j}\right)=\lambda$.

Hence, $g$ is a super set-ordered edge-magic total labelling of $G$. when $p$ is odd.

Case 2. If $p=2 \beta$, then $\| X|-| Y \mid=0$.

Let $\alpha=5 \beta n+1$. We define a labelling $h$ of $G$ as:

(i) For $k \in[1, \beta]$, set $h\left(u_{k, i}\right)=n(k-1)+1+g_{0}\left(u_{i}\right)-g_{0}\left(u_{1}\right), i \in[1$, $s] ; h\left(v_{k, j}\right)=n(\beta+k-1)+g_{0}\left(v_{j}\right)-g_{0}\left(v_{1}\right)+1, j \in[1, t]$; and for every edge $u_{k, i} v_{k, j} \in E(G)$, set $h\left(u_{k, i} v_{k, j}\right)=g_{0}\left(u_{i} v_{j}\right)+2 n(2 \beta-k)$.

(ii) For $l \in[\beta+1,2 \beta]$, set $h\left(u_{l, i}\right)=n(3 \beta+1-l)-g_{0}\left(u_{i}\right)+g_{0}\left(u_{1}\right)$, $i \in[1, s] ; h\left(v_{l, j}\right)=n(2 \beta+1-l)-g_{0}\left(v_{j}\right)+g_{0}\left(v_{1}\right), j \in[1, t] ;$ and for each edge $u_{l, i} v_{l, j} \in E(G)$, set $h\left(u_{l, i} v_{l, j}\right)=2 \ln -g_{0}\left(u_{i} v_{j}\right)$.

We show that $h$ is a super set-ordered edge-magic total labelling of $G$. When $k \in[1, \beta], i \in[1, s]$ and $j \in[1, t]$, every edge $u_{k, i} v_{k, j} \in E\left(H_{k}\right)$ holds $h\left(u_{k, i}\right)+h\left(u_{k, i} v_{k, j}\right)+h\left(v_{k, j}\right)=n(k-1)+$ $1+g_{0}\left(u_{i}\right)-g_{0}\left(u_{1}\right)+g_{0}\left(u_{i} v_{j}\right)+2 n(2 \beta-k)+n(\beta+k-1)+g_{0}\left(v_{j}\right)-g_{0}\left(v_{1}\right)+$ $1=n(5 \beta-2)-g_{0}\left(u_{1}\right)-g_{0}\left(v_{1}\right)+g_{0}\left(u_{1}\right)+g_{0}\left(v_{1}\right)+2 n-1=5 n \beta+1=\alpha$.

For $l \in[\beta+1,2 \beta], i \in[1, s]$ and $j \in[1, t]$, each edge $u_{l, i} v_{l, j} \in E\left(H_{l}\right)$ holds $h\left(u_{l, i}\right)+h\left(u_{l, i} v_{l, j}\right)+h\left(v_{l, j}\right)=n(3 \beta+1-l)-g_{0}\left(u_{i}\right)+g_{0}\left(u_{1}\right)+2 l n-$ $g_{0}\left(u_{i} v_{j}\right)+n(2 \beta+1-l)-g_{0}\left(v_{j}\right)+g_{0}\left(v_{1}\right)=n(5 \beta+2)+g_{0}\left(u_{1}\right)+g_{0}\left(v_{1}\right)-g_{0}($ $\left.u_{1}\right)-g_{0}\left(v_{1}\right)-2 n+1=5 n \beta+1=\alpha$.

Notice that $h\left(u_{k, i}\right)+h\left(u_{k, i} v_{k, j}\right)+h\left(v_{k, j}\right)=\alpha$ for $k \in[1,2 \beta]$, $i \in[1, s]$ and $j \in[1, t]$. Therefore, for $H_{k}(k \in[1,2 \beta])$ every edge $u_{k, i} v_{k, j}$, the labelling $g$ is a labelling such that $H_{k}$ holds $h\left(u_{k, i}\right)+h\left(u_{k, i} v_{k, j}\right)+h\left(v_{k, j}\right)=\alpha$ for $k \in[1,2 \beta]$.

Similarly with the case of odd $p$, for $k \in[1,2 \beta]$ and $i \in[1$, $s]$, we identify the vertex $u_{k, i} \in X_{k} \subseteq V\left(H_{k}\right)$ with the vertex $w_{k} \in V(T)$ into one vertex, and then substitute $f^{\prime}\left(w_{k}\right)$ by 
$g\left(u_{k, i}\right)$, finally, set $g\left(w_{i} w_{j}\right)=f^{\prime}\left(w_{i} w_{j}\right)$ for edges $w_{i} w_{j} \in E(T)$. On the other hand, for $k \in[1,2 \beta]$ and $j \in[1, t]$, if we identify the vertex $v_{k, j} \in Y_{k} \subseteq V\left(H_{k}\right)$ with the vertex $w_{k} \in V(T)$ into one, we replace $f^{\prime}\left(w_{k}\right)$ by $g\left(v_{k, j}\right)$, and then define $g\left(w_{i} w_{j}\right)=f^{\prime}\left(w_{i} w_{j}\right)$ for edges $w_{i} w_{j} \in E(T)$.

Here, the labelling $h$ of $G$ is defined well. We verify that $f^{\prime}\left(w_{i}\right)+f^{\prime}\left(w_{i} w_{j}\right)+f^{\prime}\left(w_{j}\right)=\alpha$ for every edge $w_{i} w_{j} \in E(T) \subseteq E(G)$.

(2.1) For a fixed $i_{0} \in[1, s]$ and $k \in[1,2 \beta]$, we identify the vertex $u_{k, i_{0}} \in X_{k} \subseteq V\left(H_{k}\right)$ with the vertex $w_{k} \in V(T)$ into one vertex. Then, for $i \in[1, \beta], j \in[\beta+1,2 \beta]$ and $l \in[1, s]$, every edge $w_{i} w_{j} \in E(T) \subseteq E(G)$ holds $f^{\prime}\left(w_{i}\right)+f^{\prime}\left(w_{i} w_{j}\right)+f^{\prime}\left(w_{j}\right)=h\left(u_{i, l}\right)$ $+h\left(w_{i} w_{j}\right)+h\left(u_{j, l}\right)=h\left(u_{i, l}\right)+n\left|f\left(w_{i}\right)-f\left(w_{j}\right)\right|+2 n \beta+h\left(u_{j, l}\right)=n(i-1)+1+$ $g_{0}\left(u_{l}\right)-g_{0}\left(u_{1}\right)+n\left|f\left(w_{i}\right)-f\left(w_{j}\right)\right|+2 n \beta+n(3 \beta+1-j)-g_{0}\left(u_{l}\right)+g_{0}\left(u_{1}\right)=n$ $(i-1+2 \beta+3 \beta+1-j)+n\left|f\left(w_{i}\right)-f\left(w_{j}\right)\right|+1=n(i+5 \beta-j)+n(j-i)+1=5 n \beta$ $+1=\alpha$.

(2.2) For a fixed $j_{0} \in[1, t]$ and $k \in[1,2 \beta]$, we identify the vertex $v_{k, j_{0}} \in Y_{k} \subseteq V\left(H_{k}\right)$ with the vertex $w_{k} \in V(T)$ into one vertex. Then, for $i \in[1, \beta], j \in[\beta+1,2 \beta]$ and $l \in[1, t]$, each edge $w_{i} w_{j} \in E(T) \subseteq E(G)$ holds $f^{\prime}\left(w_{i}\right)+f^{\prime}\left(w_{i} w_{j}\right)+f^{\prime}\left(w_{j}\right)=h\left(v_{i, l}\right)$ $+h\left(w_{i} w_{j}\right)+h\left(v_{j, l}\right)=h\left(v_{i, l}\right)+n\left|f\left(w_{i}\right)-f\left(w_{j}\right)\right|+2 n \beta+h\left(v_{j, l}\right)=n(\beta+i-1)+$ $g_{0}\left(v_{l}\right)-g_{0}\left(v_{1}\right)+1+n\left|f\left(w_{i}\right)-f\left(w_{j}\right)\right|+2 n \beta+n(2 \beta+1-j)-g_{0}\left(v_{l}\right)+g_{0}\left(v_{l}\right)=$ $\mathrm{n}(\beta+i-1)+1+n\left|f\left(w_{i}\right)-f\left(w_{j}\right)\right|+2 n \beta+n(2 \beta+1-j)=n(\beta+i-1+2 \beta+2$ $\beta+1-j)+n(j-i)+1=n(5 \beta+i-j)+n(j-i)+1=5 n \beta+1=\alpha$.

The above facts enable us to conclude that the bipartition $\left(X^{*}, Y^{*}\right)$ of $G$ holds $h\left(X^{*}\right)<h\left(Y^{*}\right)$, where $h\left(X^{*}\right)=\left\{h\left(u_{k, i}\right): k \in[1\right.$, $\beta], i \in[1, s]\} \cup\left\{h\left(v_{k, j}\right): k \in[\beta+1,2 \beta], j \in[1, t]\right\}, h\left(Y^{*}\right)=\left\{h\left(u_{k, i}\right):\right.$ $k \in[\beta+1,2 \beta], i \in[1, s]\} \cup\left\{h\left(v_{k, j}\right): k \in[1, \beta], j \in[1, t]\right\}$.

Furthermore, $h(E(G))=[2 n \beta+1,4 n \beta-1]$. Hence, $g$ is a super set-ordered edge-magic total labelling of $G$ for even $p$, as desired. The theorem follows the proof of Case 1 and Case 2.

\section{ACKNOWLEDGMENT}

This research is supported by the National Natural Science Foundation of China, Grant No. 61163054 and Grant No. 61163037; Research Projects of Graduate Teacher of Gansu University No.1216-01. We, also, are particularly indebted to Yuan Yao (Phoenix New Media, Beijing, 100029, China) for network suggestion.

\section{REFERENCES}

[1] J. A. Bondy, U. S. R. Murty. Graph Theory with Applications. The MaCmillan Press ltd, London and Basingstoke, New York, 1976.

[2] B. Yao, X.-Q. Zhou, J.-J. Zhang, te al. Labellings And Invariants Of Models From Complex Networks. to appear.

[3] Joseph A. Gallian. A Dynamic Survey of Graph Labelling. The Electronic Journal of Combinatorics, 14 (2009), \#DS6.

[4] R. B. Gnanajothi. Topics in Graph Theory. Ph. D. Thesis, Madurai Kamaraj University, 1991.

[5] M. Baca, F. Bertault, J. MacDougall, M. Miller, R. Simanjuntak, and Slamin, Vertex-antimagic total labelings of graphs, Discuss. Math. Graph Theory, 23 (2003)67-83.

[6] A. Kotzig and A. Rosa, Magic valuations of finite graphs, Canad. Math. Bull, 13 (1970), 451-461.

[7] B. Yao, H. Cheng, M. Yao, M.-M. Zhao. A Note on Strongly Graceful Trees. Ars Combinatoria 92 (2009), 155-169.
[8] Graham R J and Sloane N J A. On additive bases and harmonious graphs, Siam J Algebraic Diccrete Mathods, 1980, 29(1): 382-404.

[9] X.-Q. Zhou, B. Yao, X.-E. Chen, H.-X Tao. A proof to the oddgracefulness of all lobsters. Ars Combinatoria 103 (2012), 13-18.

[10] Xiangqian Zhou, Bing Yao, Xiang'en Chen. On Odd-gracefulness of All Symmetric Trees. to appear in JCMCC

[11] G. S. Bloom and S.W. Golomb, Applications of numbered undirected graphs, Proc. IEEE 65 (1977), 562-570.

[12] G. S. Bloom and S.W. Golomb, Numbered complete graphs, unusual rules, and assorted applications, In: Theory and Applications of Graphs, Lecture Notes in Math. 642 (1978), 53-65.

[13] A. Rosa, On certain valuations of the vertices of a graph, Theory of Graphs, Internat. Symposium, Rome, July 1966, Gordon and Breach, N.Y. and Dunod Paris (1967), 349-355.

[14] J. Sedlacek, Problem 27, In: Theory and Its Applications, Proc. Symp. Smolenice, 1963, 163-169.

[15] B. M. Stewart, Magic graphs, Can. J. Math. 18 (1966), 1031-1056.

[16] A. Kotzig and A. Rosa. Magic valuations of finite graphs. Canada. Math. Bull., 13 (1970), 451-461.

[17] H. Enomoto, A. S. Llado, T. Nakamigawa, and G. Ringel. Super edgemagic graphs. SUT J. Math., 34 (1998), 105-109.

[18] Li, L., Alderson, D., Tanaka, R., Doyle, J.C., and Willinger, W. Towards A Theory Of Scale-Free Graphs: Definition, Properties, And Implications. Internet Mathematics Vol. 2, (4) (2005), 431-523.

[19] B. Yao, X.-E. Chen, X.-Q. Zhou, J.-J. Zhang, X.-M. Zhang, M. Yao, M.-G. Li, J.-M. Xie. Graphs Related With Scale-free Networks. Second International Conference on Electronics, Communications, and Control (ICECC 2012, Zhoushan), Los Alamitos, California, Washington,Tokyo, Conference Publishing services, IEEE Computer Society, 2012: 284-287. [20] B. Yao, X.-Q. Zhou, J.-J. Zhang, X.-E. Chen, X.-M. Zhang, J.-M. Xie, M. Yao, M.-G. Li. Labellings And Invariants Of Models From Complex Networks. Proceeding of 2012 International Conference on Systems and Informa-tics, Yantai, China. IEEE catalog number: CFP1273R-CDR, ISBN: 978-1-4673-097-8.2012: 1616-1620. 